ANNALES

POLONICI MATHEMATICI

$99.2(2010)$

\title{
An improvement of Hayman's inequality on an angular domain
}

\author{
by CAI-Feng Yi (Nanchang), Yu WANG (Nanchang) and \\ HONG-YAN XU (Jingdezhen)
}

\begin{abstract}
We investigate the properties of meromorphic functions on an angular domain, and obtain a form of Yang's inequality on an angular domain by reducing the coefficients of Hayman's inequality. Moreover, we also study Hayman's inequality in different forms, and obtain accurate estimates of sums of deficiencies.
\end{abstract}

1. Introduction. We use $\mathbb{C}$ to denote the open complex plane, $\widehat{\mathbb{C}}(=$ $\mathbb{C} \cup\{\infty\})$ to denote the extended complex plane, and $\mathbb{D}(\subset \mathbb{C})$ to denote a domain. It is assumed that the reader is familiar with the notations of Nevanlinna theory such as $T(r, f), m(r, f), N(r, f), \bar{N}(r, f)$ and so on, that can be found, for instance, in [4, 11].

In [4], W. K. Hayman obtained the following well-known theorem by investigating the characteristic functions of a meromorphic function and its derivative in the complex plane.

THEOREM 1.1 (see [4]). Let $f$ be a transcendental meromorphic function on complex plane. Then for any positive integer $k$, we have

$$
T(r, f)<\left(2+\frac{1}{k}\right) N\left(r, \frac{1}{f}\right)+\left(2+\frac{2}{k}\right) N\left(r, \frac{1}{f^{(k)}-1}\right)+S(r, f),
$$

where $S(r, f)$ is a remainder term satisfying

(i) $S(r, f)=O(\log r)(r \rightarrow \infty)$ if the order of $f(z)$ is finite;

(ii) $S(r, f)=O(\log (r T(r, f)))(r \rightarrow \infty, r \notin E)$ if the order of $f(z)$ is infinite, where $E$ is a set with finite linear measure.

REMARK 1.2. Theorem 1.1 is called Hayman's inequality.

Theorem 1.3 (see [11], the Second Fundamental Theorem). Suppose that $f(z)$ is a nonconstant meromorphic function in the complex plane and

2010 Mathematics Subject Classification: Primary 30D35.

Key words and phrases: meromorphic function, angular distribution, Hayman inequality. 
$a_{1}, \ldots, a_{q}$ are $q(\geq 3)$ distinct values in the extended complex plane. Then

$$
(q-2) T(r, f)<\sum_{j=1}^{q} N\left(r, \frac{1}{f-a_{j}}\right)-N_{1}(r)+S(r, f),
$$

where $S(r, f)$ is a remainder term with the same properties as in Theorem 1.1 and $N_{1}(r)=2 N(r, f)-N\left(r, f^{\prime}\right)+N\left(r, 1 / f^{\prime}\right)$.

Remark 1.4. From Theorem 1.1, we know that the characteristic function $T(r, f)$ is controlled by only two counting functions, and without the counting function of the derivative function we cannot obtain a better conclusion than the one of Theorem 1.1. Moreover, to contrast the above two theorems, the coefficients of the counting functions in Theorem 1.1 are larger than the ones in Theorem 1.3.

In view of Remark 1.4, W. K. Hayman 4 put forward the question whether the coefficients of the counting functions $N(r, 1 / f)$ and $N\left(r, 1 / f^{(k)}-1\right)$ are best possible in Theorem 1.1. In [10, Yang further investigated the above question and established the well-known Yang inequality, in which the coefficients of the counting functions are more precise than the ones in Hayman's inequality.

THEOREM 1.5 (see [11]). Let $f$ be a transcendental meromorphic function on the complex plane. Then for any $\varepsilon>0$ and positive integer $k$, we have

$$
\begin{aligned}
T(r, f)< & \left(1+\frac{1}{k}\right) N\left(r, \frac{1}{f}\right)+\left(1+\frac{1}{k}\right) N\left(r, \frac{1}{f^{(k)}-1}\right)-N\left(r, \frac{1}{f^{(k+1)}}\right) \\
& +\varepsilon T(r, f)+S(r, f),
\end{aligned}
$$

where $S(r, f)$ is as in Theorem 1.1. Furthermore, if $a, b$ are two finite complex numbers and $b \neq 0$, then

$$
\delta(a, f)+\delta_{k}\left(b, f^{(k)}\right) \leq \frac{k+2}{k+1},
$$

where

$$
\delta(a, f)=1-\limsup _{r \rightarrow \infty} \frac{N\left(r, \frac{1}{f-a}\right)}{T(r, f)}, \quad \delta_{k}\left(a, f^{(k)}\right)=1-\limsup _{r \rightarrow \infty} \frac{N\left(r, \frac{1}{f^{(k)}-a}\right)}{T(r, f)} .
$$

It is also of interest to extend some important inequalities and results of value distribution of meromorphic functions in the whole complex plane to angular domains. Yang [10] extended Theorem 1.1 to angular domains. Recently, Zheng [13, 14], Xu and Yi [9], Xu and Cao [8, Lin [5] and others investigated the uniqueness of meromorphic functions in an angular domain and obtained some important results (see also [1, 6, 7]).

To state our results, we require the following basic notations and definitions (see [4, 13, 14]). 
Let $f$ be a meromorphic function on the angular domain $\bar{\Omega}(\alpha, \beta)=\{z$ : $\alpha \leq \arg z \leq \beta\}$ and $0<\beta-\alpha \leq 2 \pi$. Define

$$
\begin{aligned}
A_{\alpha, \beta}(r, f) & =\frac{\omega}{\pi} \int_{1}^{r}\left(\frac{1}{t^{\omega}}-\frac{t^{\omega}}{r^{2 \omega}}\right)\left\{\log ^{+}\left|f\left(t e^{i \alpha}\right)\right|+\log ^{+}\left|f\left(t e^{i \beta}\right)\right|\right\} \frac{d t}{t}, \\
B_{\alpha, \beta}(r, f) & =\frac{2 \omega}{\pi r^{\omega}} \int_{\alpha}^{\beta} \log ^{+}\left|f\left(r e^{i \theta}\right)\right| \sin \omega(\theta-\alpha) d \theta, \\
C_{\alpha, \beta}(r, f) & =2 \sum_{1<\left|b_{\mu}\right|<r}\left(\frac{1}{\left|b_{\mu}\right|^{\omega}}-\frac{\left|b_{\mu}\right|^{\omega}}{r^{2 \omega}}\right) \sin \omega\left(\theta_{\mu}-\alpha\right), \\
D_{\alpha, \beta}(r, f) & =A_{\alpha, \beta}(r, f)+B_{\alpha, \beta}(r, f), \\
S_{\alpha, \beta}(r, f) & =D_{\alpha, \beta}(r, f)+C_{\alpha, \beta}(r, f),
\end{aligned}
$$

where $\omega=\pi /(\beta-\alpha)$ and $b_{\mu}=\left|b_{\mu}\right| e^{i \theta_{\mu}}(\mu=1,2, \ldots)$ are the poles of $f$ in $\bar{\Omega}(\alpha, \beta)$ counted according to their multiplicities. $S_{\alpha, \beta}(r, f)$ is called the Nevanlinna angular characteristic, and $C_{\alpha, \beta}(r, f)$ is the angular counting function of the poles of $f$ in $\bar{\Omega}(\alpha, \beta)$; if we only consider the distinct poles of $f$, we denote the corresponding angular counting function by $\bar{C}_{\alpha, \beta}(r, f)$. Similarly, when $a \neq \infty$, we will use the notations $A_{\alpha, \beta}\left(r, \frac{1}{f-a}\right), B_{\alpha, \beta}\left(r, \frac{1}{f-a}\right)$, $C_{\alpha, \beta}\left(r, \frac{1}{f-a}\right), S_{\alpha, \beta}\left(r, \frac{1}{f-a}\right)$ and so on.

For $a \in \widehat{\mathbb{C}}$, we define

$$
\begin{gathered}
\delta_{\alpha, \beta}(a, f)=1-\limsup _{r \rightarrow \infty} \frac{C_{\alpha, \beta}\left(r, \frac{1}{f-a}\right)}{S_{\alpha, \beta}(r, f)}, \\
\delta_{\alpha, \beta}^{k}\left(a, f^{(k)}\right)=1-\limsup _{r \rightarrow \infty} \frac{C_{\alpha, \beta}\left(r, \frac{1}{f^{(k)}-a}\right)}{S_{\alpha, \beta}(r, f)} .
\end{gathered}
$$

In 1990, Yang [10] obtained the following result which extended Theorem 1.1 to angular domains.

THEOREM 1.6 (see [10]). Let $f$ be a transcendental meromorphic function on the complex plane, and $\bar{\Omega}(\alpha, \beta)$ be an angular domain. Then for any positive integer $k$, we have

$$
S_{\alpha, \beta}(r, f) \leq\left(2+\frac{1}{k}\right) C_{\alpha, \beta}\left(r, \frac{1}{f}\right)+\left(2+\frac{2}{k}\right) \bar{C}_{\alpha, \beta}\left(r, \frac{1}{f^{(k)}-1}\right)+Q_{\alpha, \beta}(r, f),
$$

where

$$
\begin{aligned}
Q_{\alpha, \beta}(r, f)= & \left(2+\frac{2}{k}\right) D_{\alpha, \beta}\left(r, \frac{f^{(k+1)}}{f^{(k)}-1}\right) \\
& +\left(2+\frac{1}{k}\right)\left[D_{\alpha, \beta}\left(r, \frac{f^{(k+1)}}{f^{(k)}}\right)+D_{\alpha, \beta}\left(r, \frac{f^{(k)}}{f}\right)\right]+O(1) .
\end{aligned}
$$


In this paper, we continue the study of meromorphic functions in angular domains and obtain the following results.

THEOREM 1.7. Let $f$ be a transcendental meromorphic function on the complex plane, and $\bar{\Omega}(\alpha, \beta)=\{z: \alpha \leq \arg z \leq \beta\}$ be an angular domain with $0<\beta-\alpha \leq 2 \pi$. Then for any $\varepsilon>0$ and positive integer $k$, we have

$$
\begin{aligned}
S_{\alpha, \beta}(r, f)< & \left(1+\frac{1}{k}\right) C_{\alpha, \beta}\left(r, \frac{1}{f}\right)+\left(1+\frac{1}{k}\right) C_{\alpha, \beta}\left(r, \frac{1}{f^{(k)}-1}\right) \\
& -C_{\alpha, \beta}\left(r, \frac{1}{f^{(k+1)}}\right)+\varepsilon S_{\alpha, \beta}(r, f)+R_{\alpha, \beta}(r, f) .
\end{aligned}
$$

Throughout, we use $R_{\alpha, \beta}(r, *)$ to denote a quantity satisfying

$$
R_{\alpha, \beta}(r, *)=O(\log (r T(r, *))), \quad r \notin E,
$$

where $E$ is a set with finite linear measure.

Furthermore, when $a, b$ are two finite complex numbers with $a \neq b$ and $b \neq 0$, and $f$ satisfies

$$
\lim _{r \rightarrow \infty} \frac{S_{\alpha, \beta}(r, f)}{\log (r T(r, f))}=\infty \quad(r \notin E)
$$

then

$$
\delta_{\alpha, \beta}(a, f)+\delta_{\alpha, \beta}^{k}\left(b, f^{(k)}\right) \leq \frac{k+2}{k+1} .
$$

Moreover, we also consider another kind of precise inequalities, and obtain an accurate estimate of the sum of deficiencies as follows.

THEOREM 1.8. Let $f$ be a transcendental meromorphic function on the complex plane, and $\bar{\Omega}(\alpha, \beta)=\{z: \alpha \leq \arg z \leq \beta\}$ an angular domain with $0<\beta-\alpha \leq 2 \pi$. Then for any finite complex numbers $a, b(a \neq b), \varepsilon>0$ and positive integer $k$, we have

$$
\begin{aligned}
S_{\alpha, \beta}\left(r, f^{(k)}\right)< & \left(1+\frac{1}{2 k}\right) C_{\alpha, \beta}\left(r, \frac{1}{f^{(k)}-a}\right)+\left(1+\frac{1}{2 k}\right) C_{\alpha, \beta}\left(r, \frac{1}{f^{(k)}-b}\right) \\
& -C_{\alpha, \beta}\left(r, \frac{1}{f^{(k)}}\right)+\varepsilon S_{\alpha, \beta}(r, f)+R_{\alpha, \beta}\left(r, f^{(k)}\right),
\end{aligned}
$$

Furthermore, if $f$ satisfies (1.1), then

$$
\delta_{\alpha, \beta}\left(a, f^{(k)}\right)+\delta_{\alpha, \beta}\left(b, f^{(k)}\right) \leq 1+\frac{1}{2 k+1} .
$$

2. Some lemmas. To prove our results, we require the following lemmas.

LEMma 2.1 (see [2]). Let $f$ be a nonconstant meromorphic function on $\bar{\Omega}(\alpha, \beta)$. Then for every complex number $a$, we have 


$$
S_{\alpha, \beta}\left(r, \frac{1}{f-a}\right)=S_{\alpha, \beta}(r, f)+\varepsilon(r, a),
$$

where $\varepsilon(r, a)=O(1)$ as $r \rightarrow \infty$.

Lemma 2.2 (see [3, p. 138]). Let $f$ be a nonconstant meromorphic function on $\mathbb{C}$ and $\bar{\Omega}(\alpha, \beta)=\{z: \alpha \leq \arg z \leq \beta\}$ be an angular domain with $0<\beta-\alpha \leq 2 \pi$. Then for any $1 \leq r<R$, we have

$$
A_{\alpha, \beta}\left(r, \frac{f^{\prime}}{f}\right) \leq K\left\{\left(\frac{R}{r}\right)^{\omega} \int_{1}^{R} \frac{\log ^{+} T(t, f)}{t^{1+\omega}} d t+\log ^{+} \frac{r}{R-r}+\log \frac{R}{r}+1\right\},
$$

and

$$
B_{\alpha, \beta}\left(r, \frac{f^{\prime}}{f}\right) \leq \frac{4 \omega}{r^{\omega}} m\left(r, \frac{f^{\prime}}{f}\right),
$$

where $\omega=\pi /(\beta-\alpha)$ and $K$ is a positive constant not depending on $r$ and $R$.

REMARK 2.3. Nevanlinna conjectured that

$$
D_{\alpha, \beta}\left(r, f^{\prime} / f\right)=A_{\alpha, \beta}\left(r, \frac{f^{\prime}}{f}\right)+B_{\alpha, \beta}\left(r, \frac{f^{\prime}}{f}\right)=o\left(S_{\alpha, \beta}\left(r, \frac{1}{f-a}\right)\right)
$$

when $r$ tends to $+\infty$ outside an exceptional set of finite linear measure, and he proved that $D_{\alpha, \beta}\left(r, f^{\prime} / f\right)=O(1)$ when the function $f$ is meromorphic in $\mathbb{C}$ and has finite order. In 1974, Gol'dberg constructed a counter-example to show that $(2.1)$ is not valid in general (see [2, 14]). However, it follows from Lemma 2.2 that

$$
D_{\alpha, \beta}\left(r, \frac{f^{\prime}}{f}\right)=A_{\alpha, \beta}\left(r, \frac{f^{\prime}}{f}\right)+B_{\alpha, \beta}\left(r, \frac{f^{\prime}}{f}\right)=R_{\alpha, \beta}(r, f),
$$

where $R_{\alpha, \beta}(r, f)=O\{\log (r T(r, f))\}$ as $r \rightarrow \infty(r \notin E)$ and $E$ is a set with finite linear measure.

LEMma 2.4 (see [15]). Let $f$ be a transcendental meromorphic function on $\mathbb{C}$, and $\bar{\Omega}(\alpha, \beta)=\{z: \alpha \leq \arg z \leq \beta\}$ be an angular domain with $0<\beta-\alpha \leq 2 \pi$. Then for any positive integer $k$, we have

$$
\begin{aligned}
S_{\alpha, \beta}(r, f) \leq & \bar{C}_{\alpha, \beta}(r, f)+C_{\alpha, \beta}\left(r, \frac{1}{f}\right)+C_{\alpha, \beta}\left(r, \frac{1}{f^{(k)}-1}\right) \\
& -C_{\alpha, \beta}\left(r, \frac{1}{f^{(k+1)}}\right)+R_{\alpha, \beta}(r, f) .
\end{aligned}
$$

Lemma 2.5. Let $f$ be a transcendental meromorphic function on $\mathbb{C}$, and $\bar{\Omega}(\alpha, \beta)=\{z: \alpha \leq \arg z \leq \beta\}$ be an angular domain with $0<\beta-\alpha \leq 2 \pi$. Then for any $\varepsilon>0$ and positive integer $k$, we have

$$
\begin{aligned}
(k-1) \bar{C}_{\alpha, \beta}(r, f)< & (1+\varepsilon) C_{\alpha, \beta}\left(r, \frac{1}{f^{(k)}}\right)+(1+\varepsilon)\left(C_{\alpha, \beta}(r, f)\right. \\
& \left.-\bar{C}_{\alpha, \beta}(r, f)\right)+R_{\alpha, \beta}(r, f) .
\end{aligned}
$$


Proof. For any given $\varepsilon>0$ and positive integer $k$, we choose a positive integer $n>k / \varepsilon$, and let $z \in \bar{\Omega}(\alpha, \beta)$. Let $W(z)=W\left(1, z, z^{2}, \ldots, z^{k+n-1}\right.$, $\left.f, z f, \ldots, z^{n} f\right)$ be the Wronskian determinant of $1, z, z^{2}, \ldots, z^{k+n-1}, f, z f$, $\ldots, z^{n} f$. We may assume $W(z) \neq 0$ because $f$ is a transcendental meromorphic function. It is easy to see that $W(z)$ is a homogeneous differential polynomial of degree $n+1$ in $f$ with polynomial coefficients of $z$ and without $f^{(j)}(j<k)$ in each term of $W(z)$.

Set $A(z)=W(z) \cdot\left(f^{(k)}(z)\right)^{-n-1}$. From Lemma 2.1, we have

$$
\begin{aligned}
C_{\alpha, \beta}(r, 1 / A) & \leq S_{\alpha, \beta}(r, A)+O(1)=C_{\alpha, \beta}(r, A)+D_{\alpha, \beta}(r, A)+O(1) \\
& \leq C_{\alpha, \beta}(r, A)+R_{\alpha, \beta}(r, f) .
\end{aligned}
$$

Now we estimate the number of zeros and poles of $A$ on $\bar{\Omega}(\alpha, \beta)$. A simple property of Wronskians gives

$$
W(z)=f^{k+2 n+1} W\left(f^{-1}, z f^{-1}, \ldots, z^{k+n-1} f^{-1}, 1, z, \ldots, z^{n}\right) .
$$

If $z_{0}$ is a pole of $f$ of order $p$, then

$$
W(z)=O\left(\left(z-z_{0}\right)^{-p(k+2 n+1)}\right), \quad z \rightarrow z_{0} .
$$

Therefore

$$
\begin{aligned}
A(z) & =O\left(\left(z-z_{0}\right)^{(n+1)(k+p)-p(k+2 n+1)}\right) \\
& =O\left(\left(z-z_{0}\right)^{n(k-1)-(k+n)(p-1)}\right)
\end{aligned}
$$

as $z \rightarrow z_{0}$.

Let $\bar{C}_{p}^{0}(r), \bar{C}_{p}^{\infty}(r)$ and $\bar{C}_{p}^{*}(r)$ be the counting functions of poles of $f$ of order $p$ on $\bar{\Omega}(\alpha, \beta)$, where $A(z)$ has a zero, pole or finite nonzero value, respectively, each pole being counted only once. From (2.2) and (2.3), we get

$$
\begin{aligned}
\sum_{p=1}^{\infty}(n(k-1)-(k+n)( & p-1)) \bar{C}_{p}^{0}(r) \\
\leq & C_{\alpha, \beta}(r, 1 / A) \leq C_{\alpha, \beta}(r, A)+R_{\alpha, \beta}(r, f) \\
\leq & \sum_{p=1}^{\infty}((k+n)(p-1)-n(k-1)) \bar{C}_{p}^{\infty}(r) \\
& +(n+1) C_{\alpha, \beta}\left(r, \frac{1}{f^{(k)}}\right)+R_{\alpha, \beta}(r, f) .
\end{aligned}
$$

If a pole of $f$ contributes to $\bar{C}_{p}^{*}(r)$, then by $(2.3)$ we get $n(k-1)-$ $(k+n)(p-1) \leq 0$ and

$$
n(k-1) \bar{C}_{p}^{*}(r) \leq(k+n)(p-1) \bar{C}_{p}^{*}(r) .
$$


Summing for $p=1,2, \ldots$ above and substituting to (2.4), we obtain

$$
\begin{aligned}
n(k-1) \sum_{p=1}^{\infty} \bar{C}_{p}(r) \leq & (k+n) \sum_{p=1}^{\infty}(p-1) \bar{C}_{p}(r) \\
& +(n+1) C_{\alpha, \beta}\left(r, \frac{1}{f^{(k)}}\right)+R_{\alpha, \beta}(r, f),
\end{aligned}
$$

where $\bar{C}_{p}(r)=\bar{C}_{p}^{0}(r)+\bar{C}_{p}^{\infty}(r)+\bar{C}_{p}^{*}(r)$.

Noting $\sum_{p=1}^{\infty}(p-1) \bar{C}_{p}(r)=\sum_{p=1}^{\infty}\left[p \bar{C}_{p}(r)-\bar{C}_{p}(r)\right]=\sum_{p=1}^{\infty}\left[C_{p}(r)-\right.$ $\left.\bar{C}_{p}(r)\right]=C_{\alpha, \beta}(r, f)-\bar{C}_{\alpha, \beta}(r, f), n>k / \varepsilon$ and (2.5), we have proved Lemma 2.5 .

LEMMA 2.6. Let $f$ be a transcendental meromorphic function on $\mathbb{C}$, and $\bar{\Omega}(\alpha, \beta)=\{z: \alpha \leq \arg z \leq \beta\}$ be an angular domain with $0<\beta-\alpha \leq 2 \pi$. Then for any $\varepsilon>0$ and positive integer $k$, we have

$$
\bar{C}_{\alpha, \beta}(r, f)<\frac{1}{k} C_{\alpha, \beta}\left(r, \frac{1}{f^{(k)}}\right)+\frac{1}{k} C_{\alpha, \beta}(r, f)+\varepsilon S_{\alpha, \beta}(r, f)+R_{\alpha, \beta}(r, f) .
$$

Proof. Replacing $\varepsilon$ with $\varepsilon / 3$ in Lemma 2.5, we have

$$
\begin{aligned}
\bar{C}_{\alpha, \beta}(r, f)< & \frac{1}{k} C_{\alpha, \beta}\left(r, \frac{1}{f^{(k)}}\right)+\frac{1}{k} C_{\alpha, \beta}(r, f)+\frac{\varepsilon}{3 k} C_{\alpha, \beta}\left(r, \frac{1}{f^{(k)}}\right) \\
& +\frac{\varepsilon}{3 k} C_{\alpha, \beta}(r, f)+R_{\alpha, \beta}(r, f) .
\end{aligned}
$$

Since

$$
\begin{aligned}
C_{\alpha, \beta}\left(r, \frac{1}{f^{(k)}}\right) & \leq S_{\alpha, \beta}\left(r, f^{(k)}\right)+O(1) \\
& \leq D_{\alpha, \beta}\left(r, \frac{f^{(k)}}{f}\right)+D_{\alpha, \beta}(r, f)+C_{\alpha, \beta}\left(r, f^{(k)}\right)+O(1) \\
& \leq D_{\alpha, \beta}(r, f)+C_{\alpha, \beta}(r, f)+k \bar{C}_{\alpha, \beta}(r, f)+R_{\alpha, \beta}(r, f) \\
& \leq(k+1) S_{\alpha, \beta}(r, f)+R_{\alpha, \beta}(r, f),
\end{aligned}
$$

from (2.7), we have

$$
\begin{aligned}
\frac{\varepsilon}{3 k} C_{\alpha, \beta}\left(r, \frac{1}{f^{(k)}}\right)+\frac{\varepsilon}{3 k} C_{\alpha, \beta}(r, f) & \leq \frac{k+2}{3 k} \varepsilon S_{\alpha, \beta}(r, f)+R_{\alpha, \beta}(r, f) \\
& \leq \varepsilon S_{\alpha, \beta}(r, f)+R_{\alpha, \beta}(r, f) .
\end{aligned}
$$

From (2.7) and (2.8), we get (2.6) easily.

Lemma 2.7. Let $f$ be a transcendental meromorphic function on $\mathbb{C}$, and $\bar{\Omega}(\alpha, \beta)=\{z: \alpha \leq \arg z \leq \beta\}$ be an angular domain with $0<\beta-\alpha \leq 2 \pi$. 
Then for any $\varepsilon>0$ and positive integer $k$, we have

$$
\begin{aligned}
\bar{C}_{\alpha, \beta}(r, f)< & \frac{1}{k} C_{\alpha, \beta}\left(r, \frac{1}{f}\right)+\frac{1}{k} C_{\alpha, \beta}\left(r, \frac{1}{f^{(k)}-1}\right)+\varepsilon S_{\alpha, \beta}(r, f) \\
& +R_{\alpha, \beta}(r, f) .
\end{aligned}
$$

Proof. From Lemma 2.6 we have

$$
\begin{aligned}
C_{\alpha, \beta} & \left(r, \frac{1}{f^{(k+1)}}\right) \\
& >(k+1) \bar{C}_{\alpha, \beta}(r, f)-C_{\alpha, \beta}(r, f)-\frac{k+1}{2} \varepsilon S_{\alpha, \beta}(r, f)-R_{\alpha, \beta}(r, f) .
\end{aligned}
$$

Substituting the above inequality back into Lemma 2.4, we obtain

$$
\begin{aligned}
k \bar{C}_{\alpha, \beta}(r, f)< & C_{\alpha, \beta}\left(r, \frac{1}{f}\right)+C_{\alpha, \beta}\left(r, \frac{1}{f^{(k)}-1}\right)+\left(C_{\alpha, \beta}(r, f)-S_{\alpha, \beta}(r, f)\right) \\
& +\frac{k+1}{2} \varepsilon S_{\alpha, \beta}(r, f)+R_{\alpha, \beta}(r, f) .
\end{aligned}
$$

Therefore

$$
\begin{aligned}
\bar{C}_{\alpha, \beta}(r, f)< & \frac{1}{k} C_{\alpha, \beta}\left(r, \frac{1}{f}\right)+\frac{1}{k} C_{\alpha, \beta}\left(r, \frac{1}{f^{(k)}-1}\right) \\
& +\frac{k+1}{2 k} \varepsilon S_{\alpha, \beta}(r, f)+R_{\alpha, \beta}(r, f) \\
< & \frac{1}{k} C_{\alpha, \beta}\left(r, \frac{1}{f}\right)+\frac{1}{k} C_{\alpha, \beta}\left(r, \frac{1}{f^{(k)}-1}\right)+\varepsilon^{\prime} S_{\alpha, \beta}(r, f)+R_{\alpha, \beta}(r, f) .
\end{aligned}
$$

This completes the proof of Lemma 2.7 .

From Theorem 1.4 in [10, we can deduce

LEMma 2.8. Let $f$ be a transcendental meromorphic function on $\mathbb{C}$, and $\bar{\Omega}(\alpha, \beta)=\{z: \alpha \leq \arg z \leq \beta\}$ be an angular domain with $0<\beta-\alpha \leq 2 \pi$. Then for any finite complex number $a, b(a \neq b)$, we have

$$
\begin{aligned}
S_{\alpha, \beta}(r, f) \leq & C_{\alpha, \beta}(r, f)+C_{\alpha, \beta}\left(r, \frac{1}{f-a}\right)+C_{\alpha, \beta}\left(r, \frac{1}{f-b}\right) \\
& -C_{\alpha, \beta}^{0}(r)+R_{\alpha, \beta}(r, f),
\end{aligned}
$$

where $C_{\alpha, \beta}^{0}(r)=2 C_{\alpha, \beta}(r, f)-C_{\alpha, \beta}\left(r, f^{\prime}\right)+C_{\alpha, \beta}\left(r, 1 / f^{\prime}\right)$.

Lemma 2.9. Let $f$ be a transcendental meromorphic function on $\mathbb{C}$, and $\bar{\Omega}(\alpha, \beta)=\{z: \alpha \leq \arg z \leq \beta\}$ be an angular domain with $0<\beta-\alpha \leq$ $2 \pi$. Then for any finite complex numbers $a, b(a \neq b), \varepsilon>0$ and positive integer $k$, 


$$
\begin{aligned}
\bar{C}_{\alpha, \beta}(r, f)< & \frac{1}{2 k} C_{\alpha, \beta}\left(r, \frac{1}{f^{(k)}-a}\right)+\frac{1}{2 k} C_{\alpha, \beta}\left(r, \frac{1}{f^{(k)}-b}\right) \\
& +\varepsilon S_{\alpha, \beta}(r, f)+R_{\alpha, \beta}(r, f) .
\end{aligned}
$$

Proof. By using Lemma 2.8 for $f^{(k)}$ and three distinct complex numbers $a, b, \infty$, we have

$$
\begin{aligned}
S_{\alpha, \beta}\left(r, f^{(k)}\right) \leq & C_{\alpha, \beta}\left(r, f^{(k)}\right)+C_{\alpha, \beta}\left(r, \frac{1}{f^{(k)}-a}\right)+C_{\alpha, \beta}\left(r, \frac{1}{f^{(k)}-b}\right) \\
& -C_{\alpha, \beta}^{0}(r)+R_{\alpha, \beta}\left(r, f^{(k)}\right),
\end{aligned}
$$

where $C_{\alpha, \beta}^{0}(r)=2 C_{\alpha, \beta}\left(r, f^{(k)}\right)-C_{\alpha, \beta}\left(r, f^{(k+1)}\right)+C_{\alpha, \beta}\left(r, 1 / f^{(k+1)}\right)$. Thus, we get

$$
\begin{aligned}
S_{\alpha, \beta}\left(r, f^{(k)}\right) \leq & \bar{C}_{\alpha, \beta}(r, f)+C_{\alpha, \beta}\left(r, \frac{1}{f^{(k)}-a}\right)+C_{\alpha, \beta}\left(r, \frac{1}{f^{(k)}-b}\right) \\
& -C_{\alpha, \beta}\left(r, \frac{1}{f^{(k+1)}}\right)+R_{\alpha, \beta}\left(r, f^{(k)}\right) .
\end{aligned}
$$

Since $S_{\alpha, \beta}\left(r, f^{(k)}\right)=D_{\alpha, \beta}\left(r, f^{(k)}\right)+C_{\alpha, \beta}(r, f)+k \bar{C}_{\alpha, \beta}(r, f)$, by applying Lemma 2.6 for $f^{(k+1)}$ we have

$$
\begin{aligned}
C_{\alpha, \beta}\left(r, \frac{1}{f^{(k+1)}}\right)> & (k+1) \bar{C}_{\alpha, \beta}(r, f)-C_{\alpha, \beta}(r, f)-(k+1) \varepsilon S_{\alpha, \beta}(r, f) \\
& -(k+1) R_{\alpha, \beta}(r, f) .
\end{aligned}
$$

Substituting the above two inequalities back into (2.10), we get

$$
\begin{aligned}
\bar{C}_{\alpha, \beta}(r, f)< & \frac{1}{2 k} C_{\alpha, \beta}\left(r, \frac{1}{f^{(k)}-a}\right)+\frac{1}{2 k} C_{\alpha, \beta}\left(r, \frac{1}{f^{(k)}-b}\right) \\
& +\frac{k+1}{2 k} \varepsilon S_{\alpha, \beta}(r, f)+\frac{k+2}{2 k} R_{\alpha, \beta}\left(r, f^{(k)}\right) \\
< & \frac{1}{2 k} C_{\alpha, \beta}\left(r, \frac{1}{f^{(k)}-a}\right)+\frac{1}{2 k} C_{\alpha, \beta}\left(r, \frac{1}{f^{(k)}-b}\right) \\
& +\varepsilon^{\prime} S_{\alpha, \beta}(r, f)+R_{\alpha, \beta}\left(r, f^{(k)}\right) .
\end{aligned}
$$

From the definition of $R_{\alpha, \beta}(r, *)$ and $T(r, f) \leq T\left(r, f^{(k)}\right) \leq(k+1) T(r, f)+$ $S(r, f)$, where $S(r, f)$ is as in Theorem 1.1, we get the conclusion of Lemma 2.9 .

3. Proof of Theorem 1.7. From Lemmas 2.4 and 2.7, we get

$$
\begin{aligned}
S_{\alpha, \beta}(r, f)< & \left(1+\frac{1}{k}\right) C_{\alpha, \beta}\left(r, \frac{1}{f}\right)+\left(1+\frac{1}{k}\right) C_{\alpha, \beta}\left(r, \frac{1}{f^{(k)}-1}\right) \\
& -C_{\alpha, \beta}\left(r, \frac{1}{f^{(k+1)}}\right)+\varepsilon S_{\alpha, \beta}(r, f)+R_{\alpha, \beta}(r, f) .
\end{aligned}
$$


Next, we prove the inequality for the sum of deficiencies. First, using the above inequality for the function $(f-a) / b$, we get

$$
\begin{aligned}
S_{\alpha, \beta}(r, f)< & \left(1+\frac{1}{k}\right) C_{\alpha, \beta}\left(r, \frac{1}{f-a}\right)+\left(1+\frac{1}{k}\right) C_{\alpha, \beta}\left(r, \frac{1}{f^{(k)}-b}\right) \\
& -C_{\alpha, \beta}\left(r, \frac{1}{f^{(k+1)}}\right)+\varepsilon S_{\alpha, \beta}(r, f)+R_{\alpha, \beta}(r, f) \\
< & \left(1+\frac{1}{k}\right) C_{\alpha, \beta}\left(r, \frac{1}{f-a}\right)+\left(1+\frac{1}{k}\right) C_{\alpha, \beta}\left(r, \frac{1}{f^{(k)}-b}\right) \\
& +\varepsilon S_{\alpha, \beta}(r, f)+R_{\alpha, \beta}(r, f) .
\end{aligned}
$$

Dividing both sides by $S_{\alpha, \beta}(r, f)$, we have

$$
\begin{aligned}
\left(1+\frac{1}{k}\right)\left(1-\frac{C_{\alpha, \beta}\left(r, \frac{1}{f-a}\right)}{S_{\alpha, \beta}(r, f)}+1-\right. & \left.\frac{C_{\alpha, \beta}\left(r, \frac{1}{f^{(k)}-b}\right)}{S_{\alpha, \beta}(r, f)}\right) \\
& <1+\frac{2}{k}+\varepsilon+\frac{R_{\alpha, \beta}(r, f)}{S_{\alpha, \beta}(r, f)} .
\end{aligned}
$$

From (3.1) and the definitions of $\delta_{\alpha, \beta}(a, f), \delta_{\alpha, \beta}^{k}\left(a, f^{(k)}\right)$, we get

$$
\begin{gathered}
\left(1+\frac{1}{k}\right)\left(\delta_{\alpha, \beta}(a, f)+\delta_{\alpha, \beta}^{k}\left(b, f^{(k)}\right)\right) \\
\leq\left(1+\frac{1}{k}\right) \liminf _{r \rightarrow \infty}\left(1-\frac{C_{\alpha, \beta}\left(r, \frac{1}{f-a}\right)}{S_{\alpha, \beta}(r, f)}+1-\frac{C_{\alpha, \beta}\left(r, \frac{1}{f^{(k)}-b}\right)}{S_{\alpha, \beta}(r, f)}\right) \\
\leq \limsup _{r \rightarrow \infty}\left(1+\frac{2}{k}+\varepsilon\right)+\liminf _{r \rightarrow \infty} \frac{R_{\alpha, \beta}(r, f)}{S_{\alpha, \beta}(r, f)} .
\end{gathered}
$$

From (1.1), we get

$$
\lim _{r \rightarrow \infty} \frac{R_{\alpha, \beta}(r, f)}{S_{\alpha, \beta}(r, f)}=0 .
$$

From (3.2), since $\varepsilon$ is arbitrary we get

$$
\delta_{\alpha, \beta}(a, f)+\delta_{\alpha, \beta}^{k}\left(b, f^{(k)}\right) \leq \frac{k+2}{k+1} .
$$

Thus, we have completed the proof of Theorem 1.7.

4. Proof of Theorem 1.8. From Lemma 2.9 and (2.10), we get

$$
\begin{aligned}
S_{\alpha, \beta}\left(r, f^{(k)}\right) \leq & \left(1+\frac{1}{2 k}\right) C_{\alpha, \beta}\left(r, \frac{1}{f^{(k)}-a}\right)+\left(1+\frac{1}{2 k}\right) C_{\alpha, \beta}\left(r, \frac{1}{f^{(k)}-b}\right) \\
& -C_{\alpha, \beta}\left(r, \frac{1}{f^{(k+1)}}\right)+\varepsilon S_{\alpha, \beta}(r, f)+R_{\alpha, \beta}\left(r, f^{(k)}\right) .
\end{aligned}
$$


The above inequality implies

$$
\begin{aligned}
\left(1+\frac{1}{2 k}\right)\left(2-\frac{C_{\alpha, \beta}\left(r, \frac{1}{f^{(k)}-a}\right)}{S_{\alpha, \beta}\left(r, f^{(k)}\right)}-\frac{C_{\alpha, \beta}\left(r, \frac{1}{f^{(k)}-b}\right)}{S_{\alpha, \beta}\left(r, f^{(k)}\right)}\right) & \\
& <1+\frac{1}{k}+\varepsilon+\frac{R_{\alpha, \beta}\left(r, f^{(k)}\right)}{S_{\alpha, \beta}\left(r, f^{(k)}\right)} .
\end{aligned}
$$

Thus, from (4.1) and the definition of $\delta_{\alpha, \beta}(a, f)$, we get

$$
\begin{aligned}
\left(1+\frac{1}{2 k}\right)( & \left.\delta_{\alpha, \beta}\left(a, f^{(k)}\right)+\delta_{\alpha, \beta}\left(b, f^{(k)}\right)\right) \\
& \leq\left(1+\frac{1}{2 k}\right) \liminf _{r \rightarrow \infty}\left(1-\frac{C_{\alpha, \beta}\left(r, \frac{1}{f^{(k)}-a}\right)}{S_{\alpha, \beta}\left(r, f^{(k)}\right)}+1-\frac{C_{\alpha, \beta}\left(r, \frac{1}{f^{(k)}-b}\right)}{S_{\alpha, \beta}\left(r, f^{(k)}\right)}\right) \\
& \leq \limsup _{r \rightarrow \infty}\left(1+\frac{1}{k}+\varepsilon\right)+\liminf _{r \rightarrow \infty} \frac{R_{\alpha, \beta}\left(r, f^{(k)}\right)}{S_{\alpha, \beta}\left(r, f^{(k)}\right)} .
\end{aligned}
$$

From (1.1), we easily get

$$
\liminf _{r \rightarrow \infty} \frac{R_{\alpha, \beta}\left(r, f^{(k)}\right)}{S_{\alpha, \beta}\left(r, f^{(k)}\right)}=0 .
$$

From (4.2), since $\varepsilon$ is arbitrary,

$$
\delta_{\alpha, \beta}\left(a, f^{(k)}\right)+\delta_{\alpha, \beta}\left(b, f^{(k)}\right) \leq 1+\frac{1}{2 k+1} .
$$

This completes the proof of Theorem 1.8.

Acknowledgements. This research was partly supported by NSF (grant no. 10871108) and the Youth Science Foundation of Jiangxi Provincial Department of Education, China (Grant No. GJJ10223).

We thank the referee(s) for reading the manuscript very carefully and making a number of valuable and kind comments which improved the presentation.

\section{References}

[1] T. B. Cao and H. X. Yi, On the uniqueness of meromorphic functions that share four values in one angular domain, J. Math. Anal. Appl. 358 (2009), 81-97.

[2] A. A. Gol'dberg, Nevanlinna's lemma on the logarithmic derivative of a meromorphic function, Math. Notes 17 (1975), 310-312.

[3] A. A. Gol'dberg and I. V. Ostrovskiı̌, The Distribution of Values of Meromorphic Functions, Nauka, Moscow, 1970 (in Russian).

[4] W. K. Hayman, Meromorphic Functions, Oxford Univ. Press, London, 1964.

[5] W. C. Lin, S. Mori and H. X. Yi, Uniqueness theorems of entire functions with shared-set in an angular domain, Acta Math. Sinica 24 (2008), 1925-1934. 
[6] Z. Q. Mao and H. F. Liu, Meromorphic functions in the unit disc that share values in an angular domain, J. Math. Anal. Appl. 359 (2009), 444-450.

[7] Z. J. Wu, A remark on uniqueness theorems in an angular domain, Proc. Japan Acad. Ser. A 64 (2008), 73-77.

[8] H. Y. Xu and T. B. Cao, Uniqueness of two analytic functions sharing four values in an angular domain, Ann. Polon. Math. 99 (2010), 55-65.

[9] J. F. Xu and H. X. Yi, On uniqueness of meromorphic functions with shared four values in some angular domains, Bull. Malays. Math. Sci. Soc. 31 (2008), 57-65.

[10] L. Yang, Precise fundamental inequalities and sums of deficiencies, Sci. China 33 (1990), 113-121.

[11] —, Value Distribution Theory, Springer/Science Press, Berlin/Beijing, 1993/1982.

[12] H. X. Yi and C. C. Yang, Uniqueness Theory of Meromorphic Functions, Science Press/Kluwer, Beijing, 2003.

[13] J. H. Zheng, On uniqueness of meromorphic functions with shared values in some angular domains, Canad. J. Math. 47 (2004), 152-160.

[14] - On uniqueness of meromorphic functions with shared values in one angular domains, Complex Var. Elliptic Equations 48 (2003), 777-785.

[15] —, Value Distribution of Meromorphic Functions, Tsinghua Univ. Press, Beijing, to appear.

Cai-Feng Yi, Yu Wang

Institute of Mathematics and Informatics

Jiangxi Normal University

330022 Nanchang, Jiangxi, P.R. China

E-mail: yicaifeng55@163.com
Hong-Yan Xu

Department of Informatics and Engineering

Jingdezhen Ceramic Institute

333403 Jingdezhen, Jiangxi, P.R. China

E-mail: xhyhhh@126.com

Received 24.9.2009

and in final form 1.7.2010 